\title{
Exotic hypernuclear systems and heavy ion collisions
}

\author{
D.E. Lanskoy ${ }^{1}$ and T.Yu. Tretyakova ${ }^{2, \star}$ \\ ${ }^{1}$ Faculty of Physics, Lomonosov Moscow State University, Moscow, 119991 Russia \\ ${ }^{2}$ Skobeltsyn Institute of Nuclear Physics, Lomonosov Moscow State University, Moscow, 119991 Russia
}

\begin{abstract}
We discuss perspectives in hypernuclear studies opened by their integration with heavy ion physics. Particularly, neutron-rich $\Lambda$ hypernuclei and double- $\Lambda$ hypernuclei can be produced, which are hardly attainable by traditional reactions and can enrich our knowledge on hadronic interactions.
\end{abstract}

\section{Introduction}

Hypernuclei are nuclear systems containing hyperon(s) in addition to nucleons. Let us start from single- $\Lambda$ hypernuclei. At present, about fifty $\Lambda$ hypernuclei from hyperhydrogen to lead are known. The first observation of a hypernucleus was made in emulsion experiments. But hypernuclear species obtained by this method are limited. The situation changed with the appearance of pionic and kaonic beams: the most detailed information on the spectra of $\Lambda$ hypernuclei was obtained by the reaction $\left(\pi^{+}, K^{+}\right)$. Also electroproduction $\left(e, e^{\prime} K^{+}\right)$is studied in the last decades.

Here we would like to discuss exotic hypernuclear systems: neutron-rich $\Lambda$ hypernuclei and hypernuclei with double strangeness.

The interest to neutron-rich hypernuclei arose in connection with the first successes in the study of light nuclei with neutron halo. Since the hyperon-nucleon interaction is attractive, hypernuclei with a stable nucleon core are more strongly bound than the corresponding core nucleus. Due to the glue-like role of the $\Lambda$ hyperon, there is a chance to stabilize loosely bound nucleon systems and even get bound hypernuclei with unstable core nucleus. Prospects for production of $\Lambda$ hypernuclei with neutron halos have been discussed by Majling [1], who has pointed out that typical neutron-rich hypernuclei, such as ${ }_{\Lambda}^{9} \mathrm{He},{ }_{\Lambda}^{11} \mathrm{Li},{ }_{\Lambda}^{12} \mathrm{Be}$, can be produced in the $\left(K^{-}, \pi^{+}\right)$reaction.

Formation of hypernuclei in experiments with heavy ions is of particular interest for hypernuclei, which cannot be obtained by mesonic or electron beams. Double-strangeness hypernuclei also can be produced in heavy ion collisions. Hypernuclei containing two hyperons ( $\Lambda \Lambda$ hypernuclei) are the main source of information about hyperon-hyperon interactions. However, in emulsion experiments to date, only few reliably identified events of the formation of $\Lambda \Lambda$ hypernuclei have been observed.

For the first time, the formation of Lambda hypernuclei in nucleus-nucleus collisions was reliably detected at LHE, JINR [2]. Later, the lightest $\Lambda$ hypernuclei were observed at GSI by the HypHI collaboration [3] at energies of several GeV/A and at high energies at RHIC [4] and LHC [5].

${ }^{\star}$ e-mail: tretyakova@sinp.msu.ru 


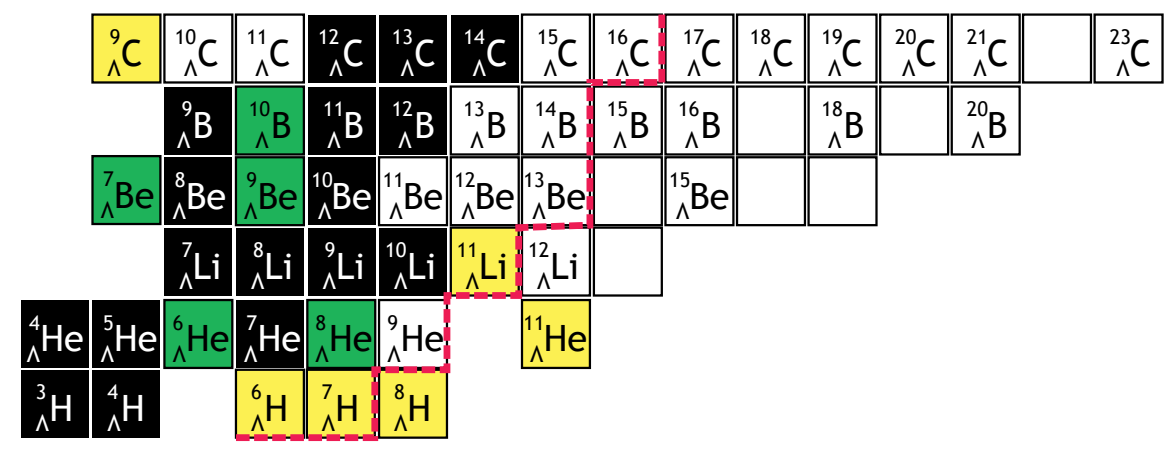

Figure 1: Chart of light $\Lambda$ hypernuclei. The black squares stand for known bound hypernuclei, which cores are also bound. The green squares correspond known bound hypernuclei, which cores are unbound. Hypernuclei shown by uncolored squares have not been observed, but evidently expected to be bound. The yellow squares label the most interesting cases: boundness of the corresponding hypernuclei is questionable and probably depends on some delicate properties of the relevant interactions. The dashed line marks the boundary of the region of hypernuclei that can be achieved in charge-exchange reactions $\left(K^{-}, \pi^{+}\right)$and $\left(\pi^{-}, K^{+}\right)$.

\section{Neutron-rich $\Lambda$ hypernuclei}

Hypernuclei with neutron excess relate to two modern fields of nuclear physics: hypernuclear studies and physics of nuclei far from the $\beta$ stability line.

Firstly, they are interesting as an example of systems with grossly extended spatial distributions, which form neutron halos in some cases. Embedding of $\Lambda$ hyperon into a halo system can examine information on its response to a perturbation. On the other hand, neutron-rich hypernuclei may allow to test hypernuclear interactions at low nuclear densities, particularly, the role of 3-body $\Lambda N N$ force or density-dependent $\Lambda N$ force can be revealed. Also, the charge symmetry breaking $\Lambda N$ interaction can be studied.

Experimentally, the neutron-rich $\Lambda$ hypernucleus $\left({ }_{\Lambda}^{10} \mathrm{Li}\right)$ was first observed at the KEK in experiment by utilizing the $\left(\pi^{-}, K^{+}\right)$double charge-exchange reaction on a ${ }^{10} \mathrm{~B}$ target [6]. The small production cross section did not allow to determine any quantitative characteristics of the hypernucleus. Evidence for the neutron-rich hypernucleus ${ }_{\Lambda}^{6} \mathrm{H}$ was presented by the FINUDA collaboration at DA $\Phi$ NE in the double charge exchange reaction at rest [7]:

$$
K_{\text {stop }}^{-}+{ }^{6} \mathrm{Li} \rightarrow{ }_{\Lambda}^{6} \mathrm{H}+\pi^{+} \text {. }
$$

Three candidate events of ${ }_{\Lambda}^{6} \mathrm{H}$ production were reported. Later an experiment to search for ${ }_{\Lambda}^{6} \mathrm{H}$ by the ${ }^{6} \mathrm{Li}\left(\pi^{-}, K^{+}\right)$reaction was carried out at J-PARC [8]. The obtained missing-mass spectrum showed no peak structure corresponding to the ${ }_{\Lambda}^{6} \mathrm{H}$ hypernucleus either below or above the ${ }_{\Lambda}^{4} \mathrm{H}+2 n$ particle decay threshold.

Which neutron-rich isotopes of hyperhydrogen are bound? This question appears to be rather nontrivial. In [9] it was pointed out that the hypernucleus ${ }_{\Lambda}^{8} \mathrm{H}$ with the unique charge-mass ratio is apparently bound. Indeed, neutron-rich hyperhydrogen isotopes may be unstable with respect to the 
Table 1: Decay modes of neutron-rich hydrogen isotopes. Data from [10]

\begin{tabular}{ccccc}
\hline Isotope & ${ }^{4} \mathrm{H}$ & ${ }^{5} \mathrm{H}$ & ${ }^{6} \mathrm{H}$ & ${ }^{7} \mathrm{H}$ \\
\hline \hline Decay mode & $n$ & $2 n$ & $3 n$ & $4 n$ \\
$E, \mathrm{MeV}$ & 3.2 & $1.7 \div 2.4$ & 2.7 & $0.57_{-21}^{+42}$ \\
\hline
\end{tabular}

neutron decay:

$$
\begin{gathered}
{ }_{\Lambda}^{6} \mathrm{H} \rightarrow{ }_{\Lambda}^{4} \mathrm{H}+n+n ; \\
{ }_{\Lambda}^{8} \mathrm{H} \rightarrow{ }_{\Lambda}^{4} \mathrm{H}+4 n .
\end{gathered}
$$

Accordingly, the neutron excess separation energy in hypernucleus $S_{X n}\left({ }_{\Lambda}^{A+1} \mathrm{Z}\right)$ is determined by the balance between the neutrons separation energy for the core $S_{X n}\left({ }^{A} \mathrm{Z}\right)$ and the difference in the hyperon binding energies $B_{\Lambda}\left({ }_{\Lambda}^{A} \mathrm{Z}\right)$ in the parent and daughter hypernuclei:

$$
\begin{gathered}
S_{2 n}\left({ }_{\Lambda}^{6} \mathrm{H}\right)=S_{2 n}\left({ }^{5} \mathrm{H}\right)+B_{\Lambda}\left({ }_{\Lambda}^{6} \mathrm{H}\right)-B_{\Lambda}\left({ }_{\Lambda}^{4} \mathrm{H}\right) ; \\
S_{4 n}\left({ }_{\Lambda}^{8} \mathrm{H}\right)=S_{4 n}\left({ }^{7} \mathrm{H}\right)+B_{\Lambda}\left({ }_{\Lambda}^{8} \mathrm{H}\right)-B_{\Lambda}\left({ }_{\Lambda}^{4} \mathrm{H}\right) .
\end{gathered}
$$

The relevant separation energies for different neutron-rich hydrogen isotopes are presented in table 1. The binding energy $B_{\Lambda}\left({ }_{\Lambda}^{4} \mathrm{H}\right)=2.0 \mathrm{MeV}$ [11]. Experimental data on light hypernuclei show that the binding energy of a hyperon strongly depends on the mass number $A$ and weakly depends on the proton-neutron ratio. So one may expect that the value of $B_{\Lambda}\left({ }_{\Lambda}^{6} \mathrm{H}\right)$ will be comparable to $B_{\Lambda}\left({ }_{\Lambda}^{6} \mathrm{He}\right)$ and is about $4 \mathrm{MeV}$. It is seen from equation (1) and Table 1 that the value of $S_{2 n}\left({ }_{\Lambda}^{6} \mathrm{H}\right)$ is close to zero. In the case of ${ }_{\Lambda}^{8} \mathrm{H}$, the situation is radically different: according to the data for ${ }_{\Lambda}^{8} \mathrm{Li}$ and ${ }_{\Lambda}^{8} \mathrm{Be}$, the $B_{\Lambda}\left({ }_{\Lambda}^{8} \mathrm{H}\right)$ should be about $7 \mathrm{MeV}$, while the separation energy $S_{4 n}\left({ }^{7} \mathrm{H}\right)$ is less than $1 \mathrm{MeV}$. From estimations (2), the hypernucleus ${ }_{\Lambda}^{8} \mathrm{H}$ should be bound and it is very important to test this in an experiment.

Theoretically, neutron-rich $\Lambda$-hypernuclei were first considered in [12]. In our work [13], the influence of a hyperon on the state of a weakly bound neutron was analyzed and it was shown that in some cases this effect is nontrivial. We considered $p$-shell $\Lambda$ hypernuclei with neutron halo in the frame of the Skyrme-Hartree-Fock approach. This method allows one to take into account the influence of hyperon not only on halo neutron(s), but also on the inner core. The neutron singleparticle energies $e_{n}$ in ${ }_{\Lambda}^{12} \mathrm{Be}$ are presented in figure 2 for various $\Lambda N$ and $N N$ interactions.

The $1 p_{1 / 2}$ halo state energy is quite sensitive to the $\Lambda N$ interaction. The most unexpected result is obtained with the strongly core-contracting SKSH1 set: hyperon addition pushes the halo state upward to the threshold despite the hyperon attraction. Moreover, if the nuclear incompressibility is low (the SkM* set), this state becomes unbound. Otherwise, the core-diluting R3 set leads to a rather tightly bound $1 p_{1 / 2}$ halo state which becomes the ground state of ${ }_{\Lambda}^{12} \mathrm{Be}$. The nonpolarizing YBZ5 set represents an intermediate case. Evidently, the halo separation energy is strongly correlated with the polarizing property of the $\Lambda N$ interaction. At the same time, the $2 s$ neutron state responds weakly to the $\Lambda N$ interaction.

Such a dependence suggests that the existence of bound hypernuclei with an unstable nucleon core, for example, ${ }_{\Lambda}^{11} \mathrm{Li}$ and ${ }_{\Lambda}^{11} \mathrm{He}$, is determined by the properties of the $\Lambda N$ interaction. As we see, while the $2 s$ state unlikely can be bound by the hyperon, the $1 p$ one possibly can. In other words, if bound ${ }_{\Lambda}^{11} \mathrm{Li}$ exists then it is most likely the $1 p$ neutron halo state, which energy is sensitive to $\Lambda N$ interaction features. The ground state of ${ }_{\Lambda}^{11} \mathrm{He}$ possibly lies just at the threshold [13].

Observing a larger number of neutron-rich $\Lambda$ hypernuclei and measuring their characteristics would be of great interest from several points of view. In addition to the natural tendency to increase the number of known hypernuclei, such hypernuclei provide information on the dependence 


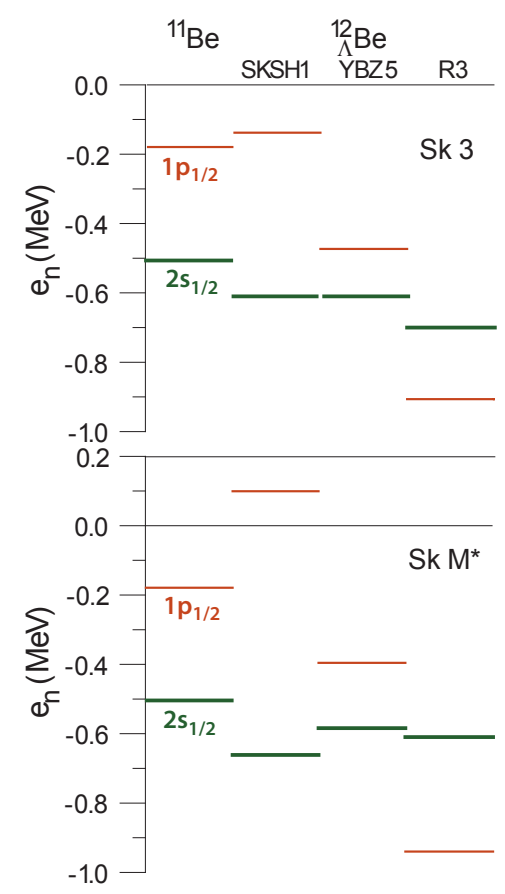

Figure 2: External neutron single-particle energies $e_{n}(\mathrm{MeV})$ of the last neutron for ${ }_{\Lambda}^{12} \mathrm{Be} . \Lambda N$ interactions are SKSH1, YBZ5, and R3. $N N$ interactions are Sk3 (upper part) and SkM*. Thick green lines are for $2 \mathrm{~s}$ states, and thin red ones are for $1 \mathrm{p}$ states. For more details, see [13].

of hyperon-nucleon interaction on density [13]. Such information is useful from the point of view of extrapolation to the region of high densities, which is valuable for both neutron star and heavy ion collision physics. Observation of neutron-rich $\Lambda$ hypernuclei is important for studying the charge symmetry breaking $\Lambda$-nucleon interaction, which was extensively discussed recently (for example, [14-16]). Till now, information on the charge symmetry breaking is extracted (with ambiguous conclusions) from the difference in the binding energies of hypernuclei $\left({ }_{\Lambda}^{4} \mathrm{H},{ }_{\Lambda}^{4} \mathrm{He}\right)$ and $\left({ }_{\Lambda}^{12} \mathrm{~B},{ }_{\Lambda}^{12} \mathrm{C}\right)$. Obviously, systems with strongly broken proton-neutron symmetry are much more informative in this regard.

\section{Double-strangeness hypernuclei}

The first event of the formation of the $\Lambda \Lambda$ hypernucleus was observed in the early 60s [17], however, so far only few more or less clearly identified events are known. In some cases, the identification of hypernuclei is ambiguous [18]. Data on $\Lambda \Lambda$ hypernuclei remain almost the only source of information about hyperon-hyperon interactions, but all that is currently known from the experiment is that the $\Lambda \Lambda$ interaction is relatively weak (by an order of magnitude weaker than between two neutrons) attraction. This conclusion is drawn from the analysis of the binding energy of the ${ }_{\Lambda \Lambda}^{6}$ He hypernucleus (the most reliable Nagara event [19]). Other data at least do not clearly contradict to this conclusion.

It is not even clear which $\Lambda \Lambda$ hypernucleus is the lightest. It is possible that this is the ${ }_{\Lambda \Lambda}^{4} \mathrm{H}$ hypernucleus, the observation of which was reported in [20]. However, this observation was called 
into question [21]. Theoretical calculations [22, 23], using almost the same input data (two-body potentials), nonetheless gave opposite answers to the question whether the system ${ }_{\Lambda \Lambda}^{4} \mathrm{H}$ is bound.

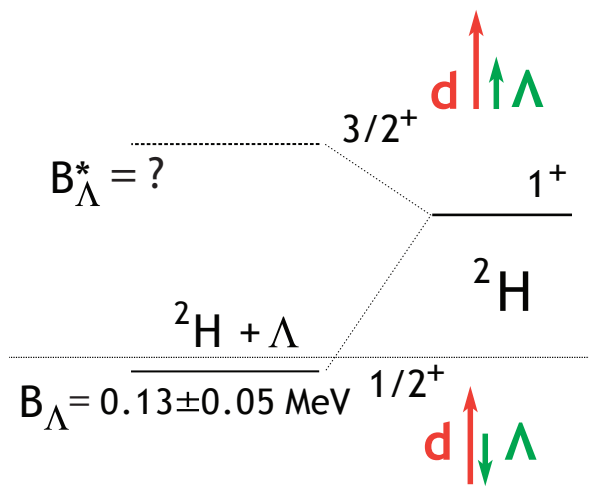

Figure 3: Level scheme of ${ }_{\Lambda}^{3} \mathrm{H}$.

Let us give a simplified description of this problem. First, neglecting the $\Lambda \Lambda$ interaction and distortion of the deuteron by the hyperons, one has:

$$
B_{\Lambda \Lambda}\left({ }_{\Lambda \Lambda}^{4} \mathrm{H}\right)=\frac{2}{3} B_{\Lambda}\left({ }_{\Lambda}^{3} \mathrm{H}_{\text {g.s. }}\right)+\frac{4}{3} B_{\Lambda}\left({ }_{\Lambda}^{3} \mathrm{H}^{*}\right),
$$

where $B_{\Lambda \Lambda}$ is the binding energy of the $\Lambda \Lambda$ pair, $B_{\Lambda}$ 's are the $\Lambda$ binding energies in the ground $\left(1 / 2^{+}\right)$ and the first excited $\left(3 / 2^{+}\right)$states of hypertriton ${ }_{\Lambda}^{3} \mathrm{H}$, respectively (see Fig. 3). Note that the latter state is probably unbound. Hereafter, $B_{\Lambda}=B_{\Lambda}\left({ }_{\Lambda}^{3} \mathrm{H}_{\text {g.s. }}\right), B_{\Lambda}^{*}=B_{\Lambda}\left({ }_{\Lambda}^{3} \mathrm{H}^{*}\right)$.

Evidently, ${ }_{\Lambda \Lambda}^{4} \mathrm{H}$ is bound if $B_{\Lambda \Lambda}>B_{\Lambda}$, or if $4 B_{\Lambda}^{*}>B_{\Lambda}$. Since $B_{\Lambda}^{*}$ is possibly negative or (if positive) very small, the last condition is unlikely fulfilled and additional $\Lambda \Lambda$ attraction may be needed to bind ${ }_{\Lambda \Lambda}^{4} \mathrm{H}$. In presence of $\Lambda \Lambda$ attraction instead (3) we have

$$
B_{\Lambda \Lambda}\left({ }_{\Lambda \Lambda}^{4} \mathrm{H}\right)=\frac{2}{3} B_{\Lambda}+\frac{4}{3} B_{\Lambda}^{*}+\delta B
$$

where $\delta B$ is the $\Lambda \Lambda$ interaction energy with the reverse sign, and a weaker condition $4 B_{\Lambda}^{*}>B_{\Lambda}-3 \delta B$. $\Lambda \Lambda$ attraction compatible with Nagara event gives $\delta B=0.15-0.2 \mathrm{MeV}$ (from [22, 23], but taking into account the updated analysis of the Nagara event in [24]) and with known $B_{\Lambda}$ we obtain that ${ }_{\Lambda \Lambda}^{4} \mathrm{H}$ is bound if $B_{\Lambda}^{*}>-0.1 \mathrm{MeV}$ (note that $B_{\Lambda}^{*}$ has not been measured yet). Summing up, boundness of ${ }_{\Lambda \Lambda}^{4} \mathrm{H}$ depends crucially on the spin dependence of the $\Lambda N$ interaction and the corresponding splitting of the spin doublet in ${ }_{\Lambda}^{3} \mathrm{H}$.

If the answer to the question above is negative, then the five-particle $\Lambda \Lambda$ hypernuclei ${ }_{\Lambda \Lambda}^{5} \mathrm{H}$ and ${ }_{\Lambda \Lambda}^{5} \mathrm{He}$ are the lightest bound. These hypernuclei have not yet been observed. Measurement of their binding energies could provide valuable information on the baryon-baryon interaction at $S=-2$. At first glance, these hypernuclei with mirror cores $\left({ }^{3} \mathrm{H}\right.$ and $\left.{ }^{3} \mathrm{He}\right)$ should have very similar properties, however, in our work [25] it was shown that the interaction energies of two hyperons in ${ }_{\Lambda \Lambda}^{5} \mathrm{H}$ and ${ }_{\Lambda \Lambda}^{5} \mathrm{He}$ can differ significantly from each other. Quantitatively, this difference is determined by the intensity of the nondiagonal interaction $\Lambda \Lambda-\Xi N$. At the same time, $\Lambda \Lambda-\Xi N$ mixing in the "next" ${ }_{\Lambda \Lambda}^{6} \mathrm{He}$ hypernucleus is not so significant. 
Various aspects of hypernuclear production in heavy-ion collisions were considered in many works, see [26-28]. Estimates [27] show that observation not only single- $\Lambda$, but also double- $\Lambda$ hypernuclei including ${ }_{\Lambda \Lambda}^{5} \mathrm{H}$ and ${ }_{\Lambda \Lambda}^{5} \mathrm{He}$ at the NICA complex is quite feasible.

\section{Conclusions, or Great Expectations}

Heavy ion beams give a unique experimental opportunity to study properties of exotic hypernuclear systems.

Neutron-rich $\Lambda$-hypernuclei (delicate properties of $\Lambda N$ interaction):

- effects of the density dependence of the $\Lambda N$ interaction;

- the response of the nucleonic system to the $\Lambda$ hyperon addition;

- the charge symmetry breaking $\Lambda N$ interaction. Hypernuclei ${ }_{\Lambda}^{8} \mathrm{H},{ }_{\Lambda}^{11} \mathrm{He},{ }_{\Lambda}^{11} \mathrm{Li}$, and ${ }_{\Lambda}^{12} \mathrm{Be}$ are of particular interest.

Double-strangeness hypernuclei (hyperonic interactions at $S=-2$ ):

- what $S=-2$ system is the lightest? Does ${ }_{\Lambda \Lambda}^{4} \mathrm{H}$ exist? Interplay of the $\Lambda \Lambda$ interaction and spin dependence of the $\Lambda N$ interaction;

- ${ }_{\Lambda \Lambda}^{5} \mathrm{H}$ and ${ }_{\Lambda \Lambda}^{5} \mathrm{He}$ : information on the $\Lambda \Lambda-\Xi N$ interaction;

- ${ }_{\Lambda \Lambda}^{6} \mathrm{He}$ : almost pure diagonal $\Lambda \Lambda$-interaction.

The hypernuclear physics is waiting for the development of experimental capabilities in the field of heavy ion collisions, in particular, for experiments at the NICA collider.

\section{References}

[1] L. Majling, Nucl. Phys. A585, 211c (1995)

[2] S.A. Avramenko et al. JETP Lett. 48, 474 (1988)

[3] T.R. Saito et al. Nucl.Phys. A954, 199 (2016)

[4] B.I. Abelev et al. Science 328, 58 (2010)

[5] J. Adam et al. Phys. Lett. B754, 360 (2016)

[6] P.K. Saha et al. Phys. Rev. Lett. 94, 052502 (2005)

[7] M. Agnello et al. Nucl. Phys. A881, 269 (2012)

[8] H. Sugimura et al. Phys. Lett. B729, 39 (2014)

[9] L. Majling, S. Gmuca. Phys. At. Nucl. 70, 1611 (2007)

[10] National Nuclear Data Center, Brookhaven, Evaluated Nuclear Structure Data File, http://ie.lbl.gov/ensdf/.

[11] A. Gal, E.V. Hungerford, D.J. Millener. Rev. Mod. Phys. 88, 035004 (2016)

[12] R.H. Dalitz, R. Levi Setti. Nuov. Cim. 30, 489 (1963)

[13] T.Yu. Tretyakova, D.E. Lanskoy. Eur. Phys. J. A5, 391 (1999)

[14] A. Gal. Phys. Lett. B744, 352 (2015)

[15] P. Achenbach. Few-Body Syst. 58, 1 (2017)

[16] E. Botta, T. Bressani, A. Felicielo. Nucl. Phys. A960, 165 (2017)

[17] M. Danycz et al. Nucl. Phys. 49, 121 (1963)

[18] K. Nakazawa et al. J. Phys. Conf. Ser. 559, 012082 (2014)

[19] H. Takahashi et al. Phys. Rev. Lett. 87, 212502 (2001) 
[20] J.K. Ahn et al. Phys. Rev. Lett. 87, 132504 (2001)

[21] S.D. Randeniya, E.V. Hungerford. Phys. Rev. C76, 064308 (2007)

[22] I.N. Filikhin, A. Gal. Phys. Rev. Lett. 89,172502 (2002).

[23] H. Nemura, Y. Akaishi, K.S. Myint. Phys. Rev. C67 051001 (2003)

[24] J.K. Ahn et al. Phys. Rev. C88, 014003 (2013)

[25] D.E. Lanskoy, Y. Yamamoto. Phys. Rev. C69, 014303 (2004)

[26] A.S. Botvina et al. Phys. Rev. C84,064904 (2011); Phys.Rev. C86, 011601 (2012); Phys. Rev.

C88, 054605 (2013); Phys. Lett. B742, 7 (2015); Eur. Phys. J. A52, 52 (2016)

[27] A. Andronic et al. Phys. Lett. B697, 293 (2011)

[28] C. Rappold, J. Lopez-Fidalgo, Phys. Rev. C94, 044616 (2016) 